\title{
3D bioprinting using stem cells
}

\author{
Chin Siang Ong ${ }^{1}$, Pooja Yesantharao', Chen Yu Huang ${ }^{2}$, Gunnar Mattson ${ }^{1}$, Joseph Boktor ${ }^{1}$, Takuma Fukunishi $^{1}$, \\ Huaitao Zhang ${ }^{1}$ and Narutoshi Hibino ${ }^{1}$
}

Recent advances have allowed for three-dimensional (3D) printing technologies to be applied to biocompatible materials, cells and supporting components, creating a field of 3D bioprinting that holds great promise for artificial organ printing and regenerative medicine. At the same time, stem cells, such as human induced pluripotent stem cells, have driven a paradigm shift in tissue regeneration and the modeling of human disease, and represent an unlimited cell source for tissue regeneration and the study of human disease. The ability to reprogram patient-specific cells holds the promise of an enhanced understanding of disease mechanisms and phenotypic variability. 3D bioprinting has been successfully performed using multiple stem cell types of different lineages and potency. The type of 3D bioprinting employed ranged from microextrusion bioprinting, inkjet bioprinting, laser-assisted bioprinting, to newer technologies such as scaffold-free spheroid-based bioprinting. This review discusses the current advances, applications, limitations and future of 3D bioprinting using stem cells, by organ systems.

S ince the first U.S. patent was awarded for 3D bioprinting (1), there has been significant advances in 3D bioprinting technology and the field of regenerative medicine (2), from bioprinting biological molecules and cells invisible to the naked eye, to biofabrication of complex tissues and organs. In terms of cell sources for bioprinting, it is now well-recognized that other than retaining the ability to recapitulate the biological function of the native tissue, it is important for bioprinted cells to be able to expand adequately for bioprinting, yet not excessively to the extent that will lead to hyperplasia or cell death. Many autologous or primary cell types, nonetheless, are difficult to isolate and culture in vitro and are limited by a finite lifespan (3).

Stem cells, on the other hand, have the properties of selfrenewal and potency, representing an unlimited cell source for $3 \mathrm{D}$ bioprinting and regenerative medicine. However, autologous adult stem cells have to be obtained invasively through bone marrow harvesting, adipose tissue extraction by liposuction or least invasively using blood apheresis. In 2006, with the discovery of human induced pluripotent stem cells (iPSCs) (4), patient-specific stem cell lines can now be created from mature cell types, such as peripheral blood mononuclear cells by venipuncture, or dermal skin fibroblasts by skin biopsy. This ability to reprogram patient-specific cells also allows for the creation of stem cells containing heritable mutations, which can then be bioprinted (5) and studied. This has the potential to improve current understanding of disease mechanisms and phenotypic variability, while minimizing rejection when transplanted back into the host for the purposes of tissue regeneration.

The purpose of this review is to examine the current advances, applications, limitations and future of 3D bioprinting using stem cells, and to a lesser degree, immortalized cell lines which are usually used for proof-of-principle bioprinting, in various organ systems (Table 1, Figure 1).

\section{D BIOPRINTING OF CARDIOVASCULAR TISSUE \\ Current Advances}

Various 3D bioprinting techniques have been applied to create 3D cardiovascular tissue constructs $(5,6)$, using different proliferative cell types. These tissue constructs exhibit synchronous macroscopic beating, and self-assembly of vessel-like conduits when co-cultured with endothelial cells (7).

Laser direct write bioprinting technology has been used to form embryoid bodies (EBs) from mouse embryonic stem cells (ESCs), and can be used to control and direct EB formation and size, allowing for directed cardiogenesis (8). Laser-Induced-Forward-Transfer (LIFT) cell bioprinting technique (9) has also been used to print mesenchymal stem cells (MSCs) and human umbilical vein endothelial cells (HUVECs) onto a cardiac patch, to promote angiogenesis and improve cardiac function, as determined by left ventricular catheterization 8 weeks after acute myocardial infarction (MI) (10).

Pneumatic extrusion 3D bioprinting technology has also been used to fabricate a tissue construct with cardiogenic potential by bioprinting human cardiac-derived cardiomyocyte progenitor cells in an alginate hydrogel scaffold (11). The 3D bioprinted cells had high cell viability, retained their commitment to the cardiac lineage and had enhanced gene expression of early cardiac transcription factors. In addition, the bioprinted cells migrated from the alginate scaffold and

${ }^{1}$ Division of Cardiac Surgery, Johns Hopkins Hospital, Baltimore, MD; ${ }^{2}$ Division of Cardiology, Johns Hopkins Hospital, Baltimore, MD. Correspondence: Narutoshi Hibino (nhibino1@jhmi.edu)

Received 18 May 2017; accepted 29 September 2017; advance online publication 1 November 2017. doi:10.1038/pr.2017.252 
fully colonized an extracellular matrix layer, forming CD31positive tubular-like structures, demonstrating that this in vitro tissue construct has the potential for in vivo function.

Table 1. Cell types used in 3D bioprinting

\begin{tabular}{|c|c|}
\hline Organ systems & Cell types used in 3D bioprinting \\
\hline Cardiovascular tissue & $\begin{array}{l}\text { ESCs }(8) \\
\text { MSCs }(10,18,19) \\
\text { Cardiac progenitor cells }(11,12,14) \\
\text { IPSC-derived cardiomyocytes }(13,20) \\
\text { Adipose-derived stromal vascular fraction } \\
\text { cells }(21) \\
\text { Myoblasts }(15,19)^{\mathrm{a}}\end{array}$ \\
\hline Musculoskeletal tissue & $\begin{array}{l}\text { MSCs (36) } \\
\text { MDSCs }(35) \\
\text { Myoblasts }(31,32)^{\mathrm{a}}\end{array}$ \\
\hline Neural tissue & $\begin{array}{l}\operatorname{ESCs}(41,42) \\
\text { MSCs }(7,43) \\
\text { Glioma stem cells }(40) \\
\operatorname{NSCs}(39,46,47)\end{array}$ \\
\hline Hepatic tissue & $\begin{array}{l}\text { iPSC-derived }(51,54) \text { or ESC-derived }(54) \\
\text { hepatocyte-like cells } \\
\text { iPSC-derived hepatic progenitor cells (57) } \\
\text { ADSCs }(57,59) \\
\text { Hepatocellular carcinoma cells }(52)^{\text {a }}\end{array}$ \\
\hline Adipose tissue & $\operatorname{ADSCs}(61)$ \\
\hline Skin tissue & $\begin{array}{l}\text { AFSCs }(65) \\
\text { MSCs }(65-67,70) \\
\text { ADSCs }(70) \\
\text { Epithelial progenitor cells }(69)\end{array}$ \\
\hline
\end{tabular}

ammortalized Cell Lines.

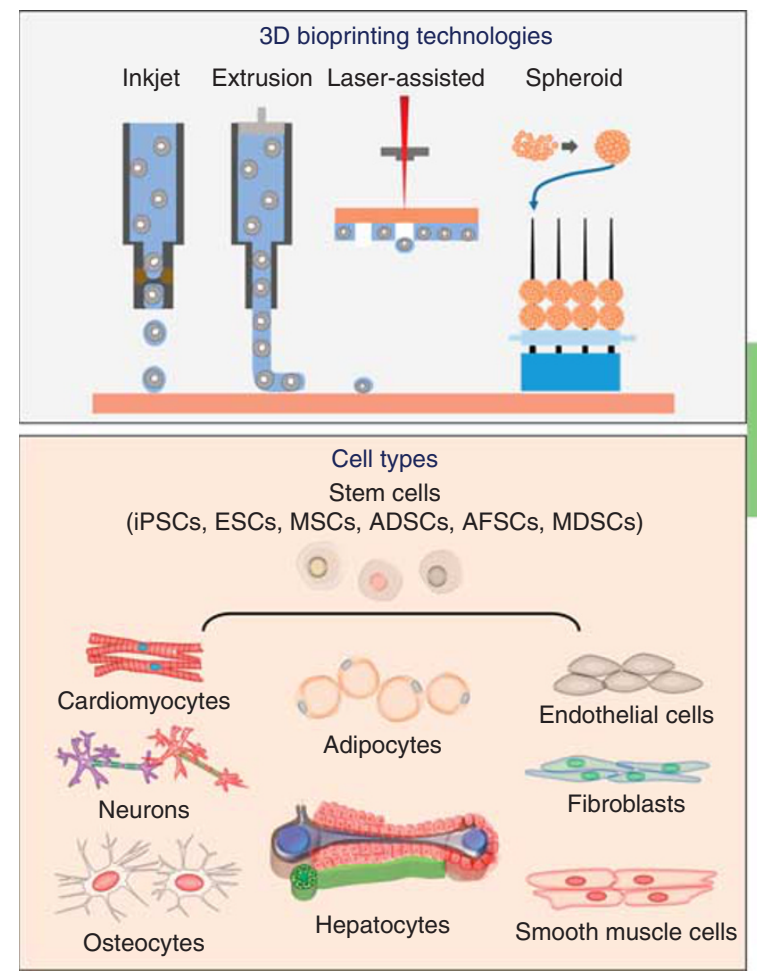

A follow-up study by this group (12) used extrusion bioprinting to bioprint cardiac progenitor cells in a gelatin/ hyaluronic acid scaffold, and the cells also retained their cardiogenic differentiation capability by increasing expression of troponin I, cardiac actinin, and connexin-43 (Cx43). This cardiac tissue construct also increased wall thickness and decreased infarct fibrosis, 4 weeks after application to the infarcted area in a mouse MI model.

In addition, extrusion bioprinting has been used to bioprint HUVEC-laden bioink (predominantly alginate, gelatin) to form a multi-layered microfibrous structure (13). The bioprinted HUVECs migrated to the peripheries of the microfibers to form a confluent layer of endothelium. This 3D endothelial construct was then seeded with human IPSCderived cardiomyocytes to fabricate aligned myocardium that could spontaneously and synchronously contract. These organoids were then embedded in microfluidic perfusion bioreactors to create an endothelialized-myocardium-on-achip platform that was used for cardiovascular toxicity evaluation (doxorubicin).

In extrusion bioprinting, bioink made using decellularized extracellular matrix derived from heart tissue has also been used. In one study (14), Vitamin B2-induced UVA crosslinking has been shown to enhance cardiomyogenic differentiation of cardiac progenitor cells, as assessed through gene expression studies and immunostaining for $\mathrm{Cx} 43$. In another study (15), Pati et al. report the use of decellularized extracellular matrix bioink derived from heart tissue and laden with myoblasts to print heart tissue constructs using extrusion bioprinting. The bioprinted constructs

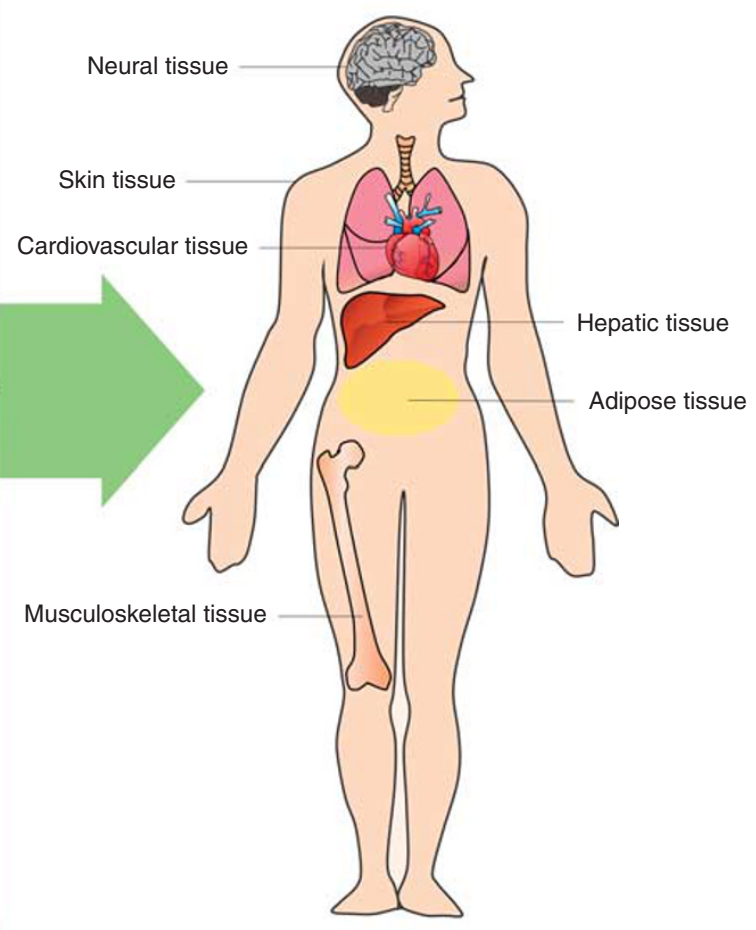

Figure 1. 3D bioprinting technologies (left top), cell types used in 3D bioprinting (left bottom), organ systems (right). 
demonstrated high cell viability and higher levels of cardiacspecific genes than those bioprinted in collagen bioinks.

Various studies have also investigated the optimization of bioinks and hydrogels for cardiac tissue bioprinting (16). One study found that time-dependent stiffening of thiolated hyaluronic acid hydrogels resulted in enhanced differentiation of primary chicken embryonic cardiomyocytes (17). Kang et al. (18) have also investigated variables associated with photocrosslinking hydrogels for their effects on extrusion bioprinting of aortic valve interstitial cells, aortic valve sinus smooth muscle cells and adipose-derived MSCs.

Transfer bioprinting has been used with specially-fabricated nanothin and highly porous membranes to bioprint highly viable, homogenous sheets of cardiomyogenically differentiated cells, derived from co-culture of MSCs and cardiomyoblast cells (19). These membranes, grafted with a thermoresponsive polymer to facilitate bioprinting, allowed for significantly enhanced differentiation of the co-cultured MSCs. This method also facilitated the formation of direct gap junctions between co-cultured cells. Repeated transferprinting on top of the first cell sheet layer was also used to fabricate multilayered sheets of differentiated cells.

Our group has also 3D bioprinted cardiac tissue using multicellular spheroids comprised of human iPSC-derived cardiomyocytes, endothelial cells, and cardiac fibroblasts. These tissue exhibit mechanical integration of spheroids and beat spontaneously (20). William et al have created human adipose stromal vascular fraction cells containing spheroids using direct-write bioprinting technology, with live/dead analysis indicating spheroid cell viability of more than $90 \%$ (21).

$3 \mathrm{D}$ bioprinting has also been used to construct heart valves, with the aim of recapitulating native valvular anatomy, but the printing materials used are usually biodegradable synthetic scaffolds and adult cell types, such as smooth muscle cells (SMCs) or valvular interstitial cells (22). Nonetheless, it may be possible to use $3 \mathrm{D}$ bioprinting, in combination with seeding using stem cells, such as autologous stem cells (bone marrow, peripheral blood) (23) or MSCs (24), to create hybrid tissue engineered heart valves.

\section{Applications}

Transplantation is currently the only viable treatment for endstage heart failure. Thus, 3D stem cell bioprinting approaches can have huge implications in regenerative medicine, for disease modeling and treatment of heart disease and heart failure, as well as for toxicological studies and personalized drug testing $(19,25)$. In fact, engineered myocardium grafts are currently in preclinical studies and may one day serve as economical and efficient solutions to MI $(7,26)$. 3D bioprinting also has potential in valvular disease, as the ability to accurately reconstruct native heart valves has enormous clinical implications, including surgical planning processes as well as regenerative medicine (22). Bioprinting cells may provide an advantage over seeding decellularized whole organs in the field of regenerative medicine, as repopulation of decellularized organs is limited by the inability to control spatial acuity and precise positioning of different cell types. 3D bioprinting technology with stem cells holds much promise when considering personalized organ regeneration (6).

\section{Limitations and Future Directions}

Heart tissue is thick and complex, and thus requires adequate vascularization and appropriate enervation to allow for functionality and biocompatibility (27). Thus, further study is needed in generating adequately vascularized heart tissue constructs of clinically-relevant thickness that can appropriately respond to electrical impulses and maintain a synchronous beating pattern, especially given that cardiomyocytes are metabolically active cells (26). This is especially a challenge given the hierarchical structure of native myocardium (13).

Heart valve engineering using 3D bioprinting often face multiple design issues and difficulties (28) and further refinement of the printing process is required to create functional and biocompatible valves. Additionally, further research into the use of stem cells in constructing heart valves through 3D bioprinting is needed, as adult cardiomyocytes and valve interstitial cells may be difficult to procure.

Silicon-nanowire field-effect transistors integrated with collagen, alginate, and PLGA scaffolds have been used to monitor the electrical activities of seeded cardiomyoctes (25). Further work should investigate the use of these nanoelectronic scaffolds (29) to provide electrical and mechanical stimulation of the cells to promote cardiomyocyte growth and stimulation. Also, further study should look into the fabrication of nextgeneration scaffolds that facilitate spontaneous beating of engineered cardiac tissue by incorporating sensing and actuating elements that allow for greater biomimicry, such as through the incorporation of carbon nanotubules in hydrogels (30), which has shown to enhance viability and phenotypical characteristics of rat ventricular myocytes (25).

\section{D BIOPRINTING OF MUSCULOSKELETAL TISSUE}

\section{Current Advances}

Various techniques have been applied to the bioprinting of musculoskeletal tissue using stem cells. $\mathrm{C}_{2} \mathrm{C}_{12}$ myoblasts, which can proliferate indefinitely and differentiate into multinucleated myotubes, has been used to fabricate 3D skeletal muscle constructs that are precisely patterned, using bioprinting techniques (27). The bioprinted cells had high viability and responded synchronously to electric pulses (31). In another study, inkjet bioprinting was used to pattern growth factors onto fibrin-coated, highly oriented sub-micron polystyrene fibers that were fabricated using a Spinneretbased Tunable Engineered parameters technique (32). This allowed for spatially-controlled, simultaneous differentiation of embryonic fibroblasts into myocyte, tenocyte, and osteoblast cell lines, creating a muscle-tendon-bone biomimetic tissue construct.

Inkjet bioprinting was also used to demonstrate simultaneous differentiation of primary muscle-derived stem cells (MDSCs) $(33,34)$ into subpopulations of osteogenic and 
myogenic cells, based on patterning of growth factor BMP-2 on fibrin-coated glass slides (35). Time-lapse microscopy was used to demonstrate multinucleated myotubule formation in MDSCs that were bioprinted off pattern, and immunocytochemistry demonstrated expression of myosin heavy chain, thus demonstrating the use of bioprinting to create spatially controlled, multi-lineage differentiation of stem cells using patterned, immobilized growth factors.

Studies have also evaluated optimal hydrogel parameters to promote myogenic differentiation of MSCs (36). Elongated focal adhesions, aligned cytoskeletons, and intermediately stiff substrates such as polydimethoxysilane that mimic native muscle textures have been shown to drive muscle tissuespecific differentiation of MSCs, as measured by upregulation of myogenic markers by gene and protein expression.

\section{Applications}

Biological microelectromechanical system devices conjugated with bioprinted skeletal muscle tissue propose promising methods of developing novel bioengineering microdevices such as motors, actuators, heart pumps, and biosensors (27). Bioprinted myotubes can also be used in muscle exercise studies, by stimulating them with electric signals at various frequencies (27). Additionally, bioprinting of musculoskeletal tissues can be used to improve material design of treatments for musculoskeletal diseases and trauma, as well as for regenerative medicine applications $(32,35)$.

\section{Limitations and Future Directions}

Myocytes maintain tissue functionality by contractile force generation, and thus bioprinted tissue must be aligned properly and must be able to recapitulate such biological function (32). Current studies have evaluated bioprinted skeletal muscle tissue conjugated with biological microelectromechanical system devices that can apply electrical impulses to the bioprinted cells, but further work is needed to evaluate the mechanical properties of bioprinted skeletal muscle tissue constructs that are of clinically-relevant thickness. Vascularization and enervation of bioprinted skeletal muscle constructs has also not yet been successfully demonstrated, and further work should evaluate strategies to effectively perfuse skeletal tissue grafts and allow for formation of functional neuromuscular junctions. One study reports printing hydrogel-based biological robots capable of spontaneous locomotion when seeded with myocytes, which the authors claim could be used to create neuromuscular junctions in vitro when combined with neurons (37).

\section{D BIOPRINTING OF NEURAL TISSUE}

\section{Current Advances}

Neurons have been bioprinted with high cell viability using inkjet and microextrusion 3D bioprinting technology, and bioprinted neurons maintained basic cellular phenotypes and functionality for over two weeks post-printing, with successful development of voltage-gated potassium and sodium channels (38). Multiple stem cell lines have been used to engineer neural tissue, including neural stem cells (NSCs), ESCs, IPSCs, adipose-derived adult stem cells and adult MSCs (39).

Glioma stem cells were used to create a glioma tumor model, as glioma stem cells are thought to be the main cell types underlying the poor prognosis associated with high grade glioma (40). The differentiation of NSCs can be directed using biologically active macromolecules and transcription factors, making these cells particularly useful when fabricating $3 \mathrm{D}$ constructs with precise spatial patterning (38). ESCs, upon formation of embryoid body cellular aggregates after printing by laser direct-write technique, demonstrate neural differentiation capacity. Neurite formation using embryoid bodies can be modulated by altering embryoid body size $(41,42)$. MSCs have been shown to preferentially differentiate towards neurogenic cells when cultured on soft matrices, as opposed to stiffer matrices that instead promote myogenic and osteogenic cell differentiation $(7,43)$.

Various studies have demonstrated the use of patterned, printed scaffolds in nerve tissue engineering (43). Linear patterns imprinted on a poly(methyl methacrylate) substrate using nano-imprinting lithography induced astrocytes to adopt a radial glia-like phenotype permissive for neural growth in vitro, without the use of additional biochemical factors (44). The patterning in the scaffolds was thought to mimic the topography of the embryonic neural stem cell niche, driving astrocytes into a radial glia-like phenotype that favors a regenerative response (44). In another study, fibronectin transfer printed on poly(lactic-co-glycolic acid) (PGLA) thin film in spatially-defined geometries was shown to upregulate markers of neurogenesis in bone marrowderived MSCs, due to altered cell morphology induced by the fibronectin-PGLA surface (45).

Multiple studies have also demonstrated the fabrication of neural tissue using 3D stem cell bioprinting approaches. $\mathrm{Gu}$ et al. bioprinted NSCs in a polysaccharide-based bioink comprised of alginate, carboxymethyl-chitosan, and agarose, using microextrusion bioprinting technology. The bioink formed 3D porous scaffolds allowing for in situ expansion and differentiation of the NSCs into functional neurons and supporting neuroglia (46). The differentiated neurons demonstrated spontaneous activity, formed synaptic contacts, established networks, had a bicuculline-induced increased calcium response, and expressed gamma-aminobutyric acid.

Inkjet bioprinting of NSCs embedded in a thermoresponsive, water-based polyurethane bioink was used to demonstrate the fabrication of bioactive scaffolds without a crosslinking agent (47). Orthotopic implantation of these 3D bioprinted neural stem cell-laden constructs was able to rescue function of adult zebrafish with traumatic brain injury, demonstrating the functionality and clinical viability of the tissue constructs. Thermal inkjet bioprinting was used to fabricate neuroglia - fibroblast growth factor-2 (FGF-2), ciliary neurotrophic factor (CNTF), and fetal bovine serum in a polyacrylamide-based hydrogel were printed into a scaffold, which was then seeded with NSCs (38). Differentiation of the NSCs was controlled using patterning of FGF-2 and CNTF, 
where increasing concentrations of CNTF resulted in differentiation of the NSCs into astrocytes expressing glial fibrillary acidic protein. Thermal inkjet bioprinting was also used to print primary rat embryonic motor neurons as well as primary rat embryonic hippocampal and cortical neurons with high viability $(38,48,49)$. The bioprinted neurons maintained their cellular phenotypes and functionality postprinting, as measured by immunostaining with dendritic marker anti-MAP2 and axonal filament anti-neurofilament NF 150, with successful development of voltage-gated potassium and sodium channels $(48,49)$. These studies also demonstrated the construction of $3 \mathrm{D}$ tissues by layering sheets of neural cells on each other using alternate inkjet printing of NT2 neuronal precursor cells and fibrin gels (48). Additionally, it was shown that $3 \mathrm{D}$ collagen gel based biopaper scaffolding decreased neurite outgrowth (49). Lee et al. demonstrated a similar layered inkjet printing technique to fabricate rat 3D neural tissue constructs. Sequential layers of collagen and rat embryonic neurons and astrocytes were bioprinted, and a solution of sodium bicarbonate was applied to each layer to allow gelation of the collagen (50). These neural cell-hydrogel composites prompted successful neurite outgrowth.

In contrast to studies demonstrating the bioprinting of normal neural tissues, Dai et al. established a glioma stem cell model using microextrusion bioprinting, as a novel tool for studying gliomagenesis and evaluating drug resistance as well as susceptibility in vitro. Glioma stem cells were encapsulated in a modified porous gelatin, alginate, and fibrinogen hydrogel that mimics the native extracellular matrix, and the bioprinted cells had an $86.92 \%$ viability (40). The glioma stem cells maintained their characteristic phenotype after printing (measured by Nestin expression) and showed differential potential (measured via glial fibrillary acidic protein and $\beta$-tubulin III markers). Furthermore, vascular endothelial growth factor was detected in the bioprinted glioma stem cells, and its expression increased during the 3week culture period, demonstrating the vascularization potential of the bioprinted tumor.

\section{Applications}

3D stem cell bioprinting of neural tissue will facilitate research into neural development, function, and disease processes, as well as translational drug screening in vitro (46). There are also possible applications in patient-specific neural tissue engineering for CNS tissue replacement following acute traumatic injury and chronic degenerative disease $(39,46)$.

As shown by Dai et al., the precise patterning achieved 3D stem cell bioprinting has a huge space of application in brain and neural tissue cancer research. This is especially important given that chemotherapy drug resistance is a huge challenge in brain tumors, largely due to brain tumor stem cells, and thus researching brain tumor stem cell biology using in vitro constructs that recapitulate the native tumor tissue microenvironment has huge potential to improve current therapeutic regimens and develop novel treatments (40). Such 3D bioprinted tumor models can also allow for replication of patients' tumors in vitro, to allow for personalization of therapies and individualized tumor testing for drug resistance and susceptibility (40).

\section{Limitations and Future Directions}

$\mathrm{Gu}$ et al. pointed towards the possibility of more expansive modeling of neuronal tissue, by developing bioinks which integrate cytokines and transcription factors that can enrich subtype neuronal expression (46,48). Further development of such bioinks could allow for the development of more complex and clinically-relevant neuronal tissue constructs. Additionally, further optimization of the hydrogel bioinks is needed for the development of thicker, more complex neural tissue structures. The lower gel modulus of hydrogels may lead to collapse if multiple layers are bioprinted, and Lee et al. demonstrated that there was reduced cell viability in thicker neuronal cell-laden collagen scaffolds $(39,50)$. Thus, the stability and viability of thicker bioprinted neural tissue must be further investigated and improved (39). The ability of the bioprinted neural tissues described above to recapitulate key physiological features of normal and diseased neural tissue models must also be further evaluated and validated before these $3 \mathrm{D}$ bioprinting approaches can be applied in a clinical setting (47).

\section{D BIOPRINTING OF HEPATIC TISSUE}

\section{Current Advances}

Various studies have looked at 3D bioprinting of liver tissue using stem cells or immortalized hepatic cell lines $(51,52)$. Stem cells in particular are studied because they have shown great promise in expressing hepatocyte-like phenotypes, and because adult hepatocytes are of limited availability, are difficult to isolate, propagate poorly, and undergo rapid functional deterioration in vitro (53).

Valve-based bioprinting was used to print iPSCs and ESCs in an alginate hydrogel matrix into structures of clinicallyrelevant thickness, and the cells were differentiated into hepatocyte-like cells (54). The bioprinted cells were positive for hepatic markers and they were phenotypically similar to native hepatocytes, as demonstrated by albumin secretion capability and morphological analysis. The bioprinted iPSCs and ESCs had negligible difference in terms of viability and pluripotency when compared to control cells, demonstrating that the bioprinting process was gentle enough to maintain the pluripotent and proliferative characteristics of the stem cells $(54,55)$. Another study demonstrated the use of a fibronectin and collagen I hydrogel embedded with hepatic growth factors that was bioprinted onto a silane-modifed glass slide to induce hepatic differentiation of seeded ESCs upon co-culture with non-parenchymal liver cells (56). Ma et al. applied 3D digital bioprinting technology to create a 3D hydrogel-based triculture model that embedded iPSC-derived hepatic progenitor cells with human umbilical vein endothelial cells and ADSCs in a microscale hexagonal architecture using gelatin methacrylate and glycidal methacrylate- 
hyalurnoic acid. This bioprinted construct showed morphological organization that recapitulated the native liver environment, as well as high levels of liver-specific gene expression, metabolic product secretion, and cytochrome P450 induction (57). Thus, this 3D biomimetic model was able to recapitulate the native liver architecture on various dimensions. Lastly, ADSCs were bioprinted alongside hepatocytes in gelatin/alginate/chitosan hydrogel, to form a vascularized liver tissue construct (58). However, the ADSCs only differentiated into spindle-shaped endothelial cells, possibly due to the short culture time, so further study is needed to develop adequately-vascularized hepatic tissue (58).

Lei and Wang have demonstrated the use of multiple nozzle 3D bioprinters with cells such as ADSCs and primary hepatocytes to fabricate complex organ precursors with branching vascular systems. Building on their current results, they also describe a potential four-nozzle low-temperature deposition manufacturing technique that could be used to generate liver organoids, using a cell-laden bioink (59).

\section{Applications}

Fabrication of liver tissue constructs and organs has enormous implications for regenerative medicine, especially given the acute need for livers and the limited numbers of viable donors (60). The creation of functional hepatic tissue also has enormous implications for personalized in vitro drug screening and liver disease studies, since the liver is one of the main sites for drug processing and excretion (57).

\section{Limitations and Future Directions}

Stem cell differentiation into hepatocytes has been demonstrated, but further work is needed to understand the molecular pathways involved in the differentiation of hepatocytes and cholangiocytes, to optimize the production of such cells needed to construct liver tissue, given the challenges with using adult hepatocytes (53). Another challenge in using engineered hepatocytes is creating metabolically appropriate conditions inside a $3 \mathrm{D}$ tissue construct, given that hepatocytes are especially metabolically active cells. Furthermore, current approaches are limited by their ability to accurately recapitulate the modular liver microenvironment, which has a complex microarchitecture and diverse cell combinations (57). Thus, further work is needed to create liver constructs that accurately model native liver tissue with its lobular architecture (58). Additional studies can look into the incorporation of diseased cell types to create more sophisticated liver disease models (57). Further work into analyzing the in vivo stability, moisture content, permeability, compatibility, and degradation rate of polymer hydrogels must also be done before 3D liver constructs can successfully be implanted in a clinical setting (59). Furthermore, current methods of 3D bioprinting with stem cells must be optimized to improve 3D viability of bioprinted liver tissue as well as vascularization of liver tissue constructs, and to facilitate higher albumin secretion of the differentiated hepatocytes $(54,58)$.

\section{D BIOPRINTING OF ADIPOSE TISSUE}

\section{Current Advances}

3D bioprinting techniques combined with stem cell technology have been used to fabricate adipose tissue. For instance, Gruene et al. used laser bioprinting techniques with human adipose-derived stem cells in alginate and blood plasma hydrogel scaffolding to fabricate $3 \mathrm{D}$ grafts. The printing methodology did not impact the proliferation ability or the differential potential of the stem cells, and the stem cells maintained their triggered lineage behavior, as measured by expression of adipogenic markers (61). Confocal microscopy was used to demonstrate that the laser bioprinting technique allowed for microscale arrangement of adipose stem cells that could allow for generating multicellular tissue grafts with a complexity similar to native adipose tissue (61). Adipose tissue constructs were also fabricated using a decellularized extracellular matrix bioink with encapsulated adipose-derived stem cells that was simultaneously bioprinted with a polycaprolactone framework (15). The decellularized extracellular matrix bioink formulation demonstrated improved cell-material interactions and adipogenesis by providing an optimized microenvironment for the bioprinted cells when compared to traditional hydrogel materials such as collagen and alginate, as demonstrated by increased expression of adipogenic markers like lipoprotein lipase (15). This study demonstrated an extrusion bioprinting technique to fabricate high-density bioprinted constructs, which has been shown to enhanced adipogenic differentiation (15).

\section{Applications}

Fabrication of biomimetic 3D adipose tissue constructs that mimic the natural architecture of adipose tissue would allow for autologous tissue replacement that could be transplanted in vivo, as well as for in vitro biomedical investigation of adipose tissue-related pathologies and in vitro drug screening $(15,61)$. In particular, the studies of adipogeneis using decellularized extracellular matrices allow for in vitro modeling of adipose tissue with more biomimetic microenvironmental architectures (15).

\section{Limitations and Future}

Adult human MSCs, which demonstrate high proliferative capability, have documented capacity to differentiate into adipocytes $(7,62)$. Thus, future work investigating the use of MSCs when 3D bioprinting adipose tissue may point towards additional sources of stem cells that can be used for adipogenesis in 3D bioprinting frameworks. Additionally, it was seen in the Gruene et al. study that thicker tissue constructs (greater than $300 \mu \mathrm{M}$ ) led to increased cell death inside the constructed $3 \mathrm{D}$ adipose tissue grafts in static culture conditions. Thus, further work is needed to improve vascularization of 3D bioprinted adipose tissue constructs, to allow for the development of viable grafts that are clinically-relevant (63). 


\section{$3 D$ bioprinting using stem cells Review}

\section{D BIOPRINTING OF SKIN}

\section{Current Advances}

The use of 3D bioprinting and stem cell technology holds much promise in skin tissue engineering. Scaffold-based tissue engineering methods have been used to reconstruct skin tissue, by seeding and cultivating cells in bioactive, printed scaffold templates (7). However, techniques that print cellladen bioinks can circumvent issues in scaffold-based tissue engineering methods, such as lack of uniform cell distributions. Furthermore, use of stem cells in these printed constructs would allow for the introduction of multiple cell types with positional specificity (7).

3D inkjet bioprinting was used to print full-thickness skin equivalents, using alternating layers of a printable and cytocompatible bioink and a dermal fibroblast cell suspension that showed high viability after printing (64). Keratinocytes were seeded onto the dermal cell construct and allowed to proliferate, and a stirring system was installed to prevent cell sedimentation (64). A noninvasive optical coherence tomography system was used to distinguish between dermal and epidermal bioprinted tissue, for in situ quality control (64).

Microextrusion bioprinting technology was used to print amniotic fluid-derived stem cells (AFSCs) and MSCs suspended in fibrin-collagen hydrogel, to investigate the use of bioprinting in augmenting wound healing in a mouse model of skin regeneration (65). The cells were bioprinted directly over the wound site, and the bioprinted construct enhanced wound closure as well as re-epithelialization as compared to controls treated with only the fibrin-collagen hydrogel. The use of AFSCs showed increased microvessel density and capillary diameters upon histological examination, as compared to MSC and control cases. However, the study showed that the bioprinted cells remained transiently and did not integrate into the newly-formed tissue, indicating that their function in wound closure and angiogenesis was a result of trophic factors they secreted. Proteomic analysis revealed that AFSCs secreted growth factors at higher concentrations than MSCs, and AFSC-conditioned media induced endothelial migration in vitro, indicating that bioprinting AFSCs could be an effective method for skin tissue regeneration for large-scale wounds and burns. Extrusion bioprinting was also used to fabricate a robust skin-like tissue, using MSCs seeded onto a printed gelatin and fibrinogen scaffold (66). The MSCs maintained a grid-like pattern even after the gel scaffold degraded, and the cells deposited a matrix that helped to form the skin-like tissue construct.

Laser bioprinting was used to fabricate a skin tissue construct, by printing functional layers of MSCs, keratinocytes, and fibroblasts $(5,38,67)$. The cells were suspended in blood plasma and alginate hydrogel, to demonstrate a computer-controlled positioning technique that can facilitate different cell types and allow for ex vivo generation of skin tissue (67). The bioprinted cells maintained $98 \%$ viability after printing, and all cell types maintained their ability to proliferate, without an increase in apoptosis or DNA fragmentation. Furthermore, the MSCs maintained their phenotype after printing, as demonstrated by fluorescence activated cell sorting analysis (67). This study used twodimensional patterning to create 3D arrangements of cell types. However, a follow-up study by the same group demonstrated the use of laser-assisted bioprinting to print fibroblasts and keratinocytes directly in 3D (68). The cells were embedded in collagen hydrogel and were used to fabricate multicellular skin grafts analogous to the native archetype, with high bioprinted cell viability and proliferation (68). The printing technology was shown to allow for precise cell localization, and the bioprinted skin grafts were shown to mimic tissue-specific functions with respect to adhering and gap junctions.

Successful regeneration of sweat glands was demonstrated using extrusion bioprinting (69). Functional, cell-laden 3D extracellular matrix mimics were fabricated in vitro using composite gelatin and sodium alginate hydrogels, and mouse plantar dermis and epidermal growth factors were synchronously incorporated into the 3D-ECM to induce mice epidermal progenitor cells. This bioprinted construct allowed for the unilateral differentiation of the epidermal progenitors into sweat glands, and direct delivery of this bioprinted construct into mice wound sites led to functional restoration of sweat glands. This study demonstrated the use of 3D bioprinting to induce specific differentiation of epidermal linages, with clinical and translational implications.

Frueh et al. reviewed vascularization strategies in skin tissue engineering- inefficient vascularization is a significant limitation of engineered tissue grafts and could lead to infection, necrosis, or loosening of implanted grafts (70). Emerging strategies in vascularization have used $3 \mathrm{D}$ bioprinting to generate highly organized microvascular networks with precise hierarchical and branching patterns. Such strategies have used 3D bioprinting techniques to assemble endothelial and parenchymal cells along with soluble trophic factors in phase-changing hydrogels, to produce vascularized constructs in a high-throughput and highly-reproducible manner (70). Of notable interest is the previously-mentioned study by Skardal et al., which demonstrated the engineering of vascularized skin tissue constructs using bioprinting techniques (65).

\section{Applications}

Engineered skin tissue has significant clinical implications. Extensive, full-thickness defects are encountered in necrotizing soft-tissue infection, trauma cases, tumor resection, and burn injuries, but autologous split-thickness skin grafts are limited and can lead to restrictive scarring (70). Engineered skin grafts have been evaluated in pre-clinical studies, and offer much promise in regenerating and repairing defective skin tissue (7). Bioprinting technologies have especially important implications for skin tissue regeneration, as this technique provides an accurate, fast manner to deliver tissue grafts directly to the wound site, as has been demonstrated in multiple mice models $(65,69)$. This is especially important 
given that skin injury and illnesses can lead to significant disability and death (71).

\section{Limitations and Future Directions}

Skin tissue bioprinting techniques using stem cells need further refinement in terms of re-creating the representative microenvironmental characteristics of native skin. The use of bioprinted vascularization techniques and sweat gland formation in skin tissue engineering is still an emerging concept, and further work is needed to advance these strategies and successfully translate them into clinical use $(65,68,69)$. Further study is also needed in the bioprinting of other skin appendages such as hair follicles, which are derived from the epidermal layer but project into the dermal layer, creating a significant challenge in terms of tissue construction (71). Multipotent skin stem cells have been identified in the hair follicle bulge at the base of the epithelial compartment, and further work should investigate patterned bioprinting of such cells to regenerate hair follicles, epidermis, and sebaceous glands in 3D skin constructs. Such work would allow for the creation of tissue constructs that better recapitulate in vivo skin architecture and composition, leading to the creation of more functional grafts.

\section{CONCLUSION}

3D bioprinting using stem cells have shown much research progress in multiple organ systems, as discussed in this review. Common challenges faced in multiple organ systems include vascularization, viability and scalability. Tissue engineering using $3 \mathrm{D}$ bioprinting technology has promising clinical potential for organ regeneration.

\section{ACKNOWLEDGMENTS}

The authors acknowledge Maricarmen Contreras, a Johns Hopkins University undergraduate student, for her help in reviewing some of the references, in the early stages of this paper.

Disclosure: The authors declare no conflict of interest.

\section{REFERENCES}

1. Boland T, Wilson WC, Xu T. Ink-jet printing of viable cells. Google Patents 2006. https://www.google.com/patents/US7051654.

2. Murphy SV, Atala A. 3D bioprinting of tissues and organs. Nature biotechnology 2014;32:773-85.

3. Dimri GP, Lee X, Basile G, et al. A biomarker that identifies senescent human cells in culture and in aging skin in vivo. Proceedings of the National Academy of Sciences 1995;92:9363-7.

4. Shi Y, Inoue $\mathrm{H}, \mathrm{Wu}$ JC, Yamanaka S. Induced pluripotent stem cell technology: a decade of progress. Nature reviews Drug discovery 2016;16: 115-30.

5. Tricomi BJ, Dias AD, Corr DT. Stem cell bioprinting for applications in regenerative medicine. Ann N Y Acad Sci 2016;1383:115-24.

6. Jung JP, Bhuiyan DB, Ogle BM. Solid organ fabrication: comparison of decellularization to 3D bioprinting. Biomater Res 2016;20:27.

7. Jakab K, Norotte C, Marga F, Murphy K, Vunjak-Novakovic G, Forgacs G. Tissue engineering by self-assembly and bio-printing of living cells. Biofabrication 2010;2:022001.

8. Bauwens CL, Peerani R, Niebruegge S, et al. Control of Human Embryonic Stem Cell Colony and Aggregate Size Heterogeneity Influences Differentiation Trajectories. STEM CELLS 2008;26:2300-10.
9. Koch L, Kuhn S, Sorg H, et al. Laser printing of skin cells and human stem cells. Tissue Eng Part C Methods 2010;16:847-54.

10. Gaebel R, Ma N, Liu J, et al. Patterning human stem cells and endothelial cells with laser printing for cardiac regeneration. Biomaterials 2011;32: 9218-30.

11. Gaetani R, Doevendans PA, Metz CH, et al. Cardiac tissue engineering using tissue printing technology and human cardiac progenitor cells. Biomaterials 2012;33:1782-90.

12. Gaetani R, Feyen DAM, Verhage V, et al. Epicardial application of cardiac progenitor cells in a 3D-printed gelatin/hyaluronic acid patch preserves cardiac function after myocardial infarction. Biomaterials 2015;61: $339-48$.

13. Zhang YS, Arneri A, Bersini S, et al. Bioprinting 3D microfibrous scaffolds for engineering endothelialized myocardium and heart-on-achip. Biomaterials 2016;110:45-59.

14. Jang J, Kim TG, Kim BS, Kim SW, Kwon SM, Cho DW. Tailoring mechanical properties of decellularized extracellular matrix bioink by vitamin B2-induced photo-crosslinking. Acta Biomater 2016;33:88-95.

15. Pati F, Jang J, Ha DH, et al. Printing three-dimensional tissue analogues with decellularized extracellular matrix bioink. Nat Commun 2014;5: 3935.

16. Prestwich GD. Hyaluronic acid-based clinical biomaterials derived for cell and molecule delivery in regenerative medicine. J Control Release 2011;155:193-9.

17. Young JL, Engler AJ. Hydrogels with time-dependent material properties enhance cardiomyocyte differentiation in vitro. Biomaterials 2011;32: 1002-9.

18. Kang LH, Armstrong PA, Lee LJ, Duan B, Kang KH, Butcher JT. Optimizing Photo-Encapsulation Viability of Heart Valve Cell Types in 3D Printable Composite Hydrogels. Ann Biomed Eng 2017;45: 360-77.

19. Ryu S, Yoo J, Jang Y, et al. Nanothin Coculture Membranes with Tunable Pore Architecture and Thermoresponsive Functionality for TransferPrintable Stem Cell-Derived Cardiac Sheets. ACS Nano 2015;9:10186-202.

20. Ong CS, Fukunishi T, Nashed A, et al. Creation of Cardiac Tissue Exhibiting Mechanical Integration of Spheroids Using 3D Bioprinting. J Vis Exp. 2017 (doi:10.3791/55438).

21. Williams SK, Touroo JS, Church KH, Hoying JB. Encapsulation of adipose stromal vascular fraction cells in alginate hydrogel spheroids using a direct-write three-dimensional printing system. Biores Open Access 2013;2:448-54.

22. Cheung DY, Duan B, Butcher JT. Current progress in tissue engineering of heart valves: multiscale problems, multiscale solutions. Expert Opin Biol Ther 2015;15:1155-72.

23. Schmidt D, Dijkman PE, Driessen-Mol A, et al. Minimally-invasive implantation of living tissue engineered heart valves: a comprehensive approach from autologous vascular cells to stem cells. Journal of the American College of Cardiology 2010;56:510-20.

24. Dijkman PE, Driessen-Mol A, Frese L, Hoerstrup SP, Baaijens FP. Decellularized homologous tissue-engineered heart valves as off-the-shelf alternatives to xeno- and homografts. Biomaterials 2012;33:4545-54.

25. Bajaj P, Schweller RM, Khademhosseini A, West JL, Bashir R. 3D biofabrication strategies for tissue engineering and regenerative medicine. Annu Rev Biomed Eng 2014;16:247-76.

26. Xin Y, Chai G, Zhang T, et al. Analysis of multiple types of human cells subsequent to bioprinting with electrospraying technology. Biomed Rep 2016;5:723-30.

27. Gao G, Cui X. Three-dimensional bioprinting in tissue engineering and regenerative medicine. Biotechnol Lett 2016;38:203-11.

28. Jana S, Lerman A. Bioprinting a cardiac valve. Biotechnology Advances 2015;33:1503-21.

29. Tian B, Liu J, Dvir T, et al. Macroporous nanowire nanoelectronic scaffolds for synthetic tissues. Nat Mater 2012;11:986-4.

30. Shin SR, Jung SM, Zalabany M, et al. Carbon-Nanotube-Embedded Hydrogel Sheets for Engineering Cardiac Constructs and Bioactuators. ACS Nano 2013;7:2369-80. 


\section{$3 D$ bioprinting using stem cells ReVieW}

31. Cui X, Gao G, Qiu Y. Accelerated myotube formation using bioprinting technology for biosensor applications. Biotechnology Letters 2013;35:315-21.

32. Ker ED, Nain AS, Weiss LE, et al. Bioprinting of growth factors onto aligned sub-micron fibrous scaffolds for simultaneous control of cell differentiation and alignment. Biomaterials 2011;32:8097-107.

33. Deasy BM, Gharaibeh BM, Pollett JB, et al. Long-term self-renewal of postnatal muscle-derived stem cells. Molecular biology of the cell 2005;16:3323-33.

34. Qu-Petersen Z, Deasy B, Jankowski R, et al. Identification of a novel population of muscle stem cells in mice: potential for muscle regeneration. The Journal of cell biology 2002;157:851-64.

35. Phillippi JA, Miller E, Weiss L, Huard J, Waggoner A, Campbell P. Microenvironments engineered by inkjet bioprinting spatially direct adult stem cells toward muscle- and bone-like subpopulations. Stem Cells 2008;26:127-34.

36. Yu H, Tay CY, Pal M, et al. A bio-inspired platform to modulate myogenic differentiation of human mesenchymal stem cells through focal adhesion regulation. Adv Healthc Mater 2013;2:442-9.

37. Chan V, Park K, Collens MB, Kong H, Saif TA, Bashir R. Development of Miniaturized Walking Biological Machines. Scientific Reports 2012;2:857.

38. Tasoglu S, Demirci U. Bioprinting for stem cell research. Trends Biotechnol 2013;31:10-9.

39. Hsieh FY, Hsu SH. 3D bioprinting: A new insight into the therapeutic strategy of neural tissue regeneration. Organogenesis 2015;11:153-8.

40. Dai X, Ma C, Lan Q, Xu T. 3D bioprinted glioma stem cells for brain tumor model and applications of drug susceptibility. Biofabrication 2016;8:045005.

41. Dias AD, Unser AM, Xie Y, Chrisey DB, Corr DT. Generating sizecontrolled embryoid bodies using laser direct-write. Biofabrication 2014;6:025007.

42. Choi YY, Chung BG, Lee DH, Khademhosseini A, Kim JH, Lee SH. Controlled-size embryoid body formation in concave microwell arrays. Biomaterials 2010;31:4296-303.

43. Lin X, Shi Y, Cao Y, Liu W. Recent progress in stem cell differentiation directed by material and mechanical cues. Biomed Mater 2016;11:014109.

44. Mattotti M, Alvarez Z, Ortega JA, Planell JA, Engel E, Alcantara S. Inducing functional radial glia-like progenitors from cortical astrocyte cultures using micropatterned PMMA. Biomaterials 2012;33:1759-70.

45. Tay CY, Yu H, Pal M, et al. Micropatterned matrix directs differentiation of human mesenchymal stem cells towards myocardial lineage. Exp Cell Res 2010;316:1159-68.

46. Gu Q, Tomaskovic-Crook E, Lozano R, et al. Functional 3D Neural MiniTissues from Printed Gel-Based Bioink and Human Neural Stem Cells. Adv Healthc Mater 2016;5:1429-38.

47. Hsieh FY, Lin HH, Hsu SH. 3D bioprinting of neural stem cell-laden thermoresponsive biodegradable polyurethane hydrogel and potential in central nervous system repair. Biomaterials 2015;71:48-57.

48. Xu T, Gregory CA, Molnar P, et al. Viability and electrophysiology of neural cell structures generated by the inkjet printing method. Biomaterials 2006;27:3580-8.

49. Xu T, Jin J, Gregory C, Hickman JJ, Boland T. Inkjet printing of viable mammalian cells. Biomaterials 2005;26:93-9.

50. Lee W, Pinckney J, Lee V, et al. Three-dimensional bioprinting of rat embryonic neural cells. Neuroreport 2009;20:798-803.

51. JB R, V G, P M, BR S, SC. P. A novel in vitro three-dimensional bioprinted liver tissue system for drug development. FASEB journal: official publication of the Federation of American Societies for Experimental Biology 2013;27(Meeting Abstract Supplement):872.12.

52. Robert C, Kamal E, Honglu W, Wei S. Biofabrication of a threedimensional liver micro-organ as an in vitro drug metabolism model. Biofabrication 2010;2:045004.

53. Lee SY, Kim HJ, Choi D. Cell sources, liver support systems and liver tissue engineering: alternatives to liver transplantation. Int J. Stem Cells 2015;8:36-47.

54. Faulkner-Jones A, Fyfe C, Cornelissen DJ, et al. Bioprinting of human pluripotent stem cells and their directed differentiation into hepatocytelike cells for the generation of mini-livers in 3D. Biofabrication 2015;7: 044102.

55. Waddington SN, Privolizzi R, Karda R, O'Neill HC. A Broad Overview and Review of CRISPR-Cas Technology and Stem Cells. Curr Stem Cell Rep 2016;2:9-20.

56. Tuleuova N, Lee JY, Lee J, Ramanculov E, Zern MA, Revzin A. Using growth factor arrays and micropatterned co-cultures to induce hepatic differentiation of embryonic stem cells. Biomaterials 2010;31:9221-31.

57. Ma X, Qu X, Zhu W, et al. Deterministically patterned biomimetic human iPSCderived hepatic model via rapid 3D bioprinting. PNAS 2016;113:2206-11.

58. Wust S, Muller R, Hofmann S. Controlled Positioning of Cells in Biomaterials-Approaches Towards 3D Tissue Printing. J Funct Biomater 2011;2:119-54

59. Lei M, Wang X. Biodegradable Polymers and Stem Cells for Bioprinting. Molecules 2016;21.pii:E539.

60. Zhou Y. The Application of Ultrasound in 3D Bio-Printing. Molecules 2016;21. pii:E590.

61. Gruene M, Pflaum M, Deiwick A, et al. Adipogenic differentiation of laser-printed 3D tissue grafts consisting of human adipose-derived stem cells. Biofabrication 2011;3:015005.

62. Yang J, Shrike Zhang Y, Yue K, Khademhosseini A. Cell-Laden Hydrogels for Osteochondral and Cartilage Tissue Engineering. Acta Biomater 2017;57:1-25.

63. Lovett M, Lee K, Edwards A, Kaplan DL. Vascularization Strategies for Tissue Engineering. Tissue Engineering 2009;15:353-70.

64. Rasch A. Fifth Congress of Industrial Cell Technology 2014. Regenerative Medicine 2014;10:105-7.

65. Skardal A, Mack D, Kapetanovic E, et al. Bioprinted amniotic fluidderived stem cells accelerate healing of large skin wounds. Stem Cells Transl Med 2012;1:792-802.

66. Rutz AL, Hyland KE, Jakus AE, Burghardt WR, Shah RN. A multimaterial bioink method for $3 \mathrm{D}$ printing tunable, cell-compatible hydrogels. Adv Mater 2015;27:1607-4.

67. Koch L, S. K, Sorg H, et al. Laser Printing of Skin Cells and Human Stem Cells. Tissue Engineering 2010;16:847-54.

68. Koch L, Deiwick A, Schlie S, et al. Skin tissue generation by laser cell printing. Biotechnol Bioeng 2012;109:1855-63.

69. Huang S, Yao B, Xie J, Fu X. 3D bioprinted extracellular matrix mimics facilitate directed differentiation of epithelial progenitors for sweat gland regeneration. Acta Biomater 2016;32:170-7.

70. Frueh FS, Menger MD, Lindenblatt N, Giovanoli P, Laschke MW. Current and emerging vascularization strategies in skin tissue engineering. Crit Rev Biotechnol 2016;37:613-25.

71. Priya SG, Jungvid H, Kumar A. Skin tissue engineering for tissue repair and regeneration. Tissue Eng Part B Rev 2008;14:105-8. 